\title{
Assessment of Different Sensor Configurations for Collaborative Driving in Urban Environments
}

\author{
Mark G. Petovello, ${ }^{1}$ Kyle O'Keefe, ${ }^{1}$ Phil Wei, ${ }^{1}$ and Chaminda Basnayake ${ }^{2}$ \\ ${ }^{1}$ Department of Geomatics Engineering, Schulich School of Engineering, University of Calgary, Canada \\ ${ }^{2}$ Electrical and Controls Integration (ECI) Laboratory, General Motors Technical Centre, USA \\ Correspondence should be addressed to Mark G. Petovello; mark.petovello@ucalgary.ca
}

Received 3 September 2012; Accepted 7 January 2013

Academic Editor: Yuei-An Liou

Copyright ( 2013 Mark G. Petovello et al. This is an open access article distributed under the Creative Commons Attribution License, which permits unrestricted use, distribution, and reproduction in any medium, provided the original work is properly cited.

\begin{abstract}
Vehicle-to-vehicle relative navigation of a network of vehicles travelling in an urban canyon is assessed using least-squares and Kalman filtering covariance simulation techniques. Between-vehicle differential GPS is compared with differential GPS augmented with between-vehicle ultrawideband range and bearing measurements. The three measurement types are combined using both least-squares and Kalman filtering to estimate the horizontal positions of a network of vehicles travelling in the same direction on a road in a simulated urban canyon. The number of vehicles participating in the network is varied between two and nine while the severity of the urban canyon was varied from 15-to 65-degree elevation mask angles. The effect of each vehicle's azimuth being known a priori, or unknown is assessed. The resulting relative positions in the network of vehicles are then analysed in terms of horizontal accuracy and statistical reliability of the solution. The addition of both range and bearing measurements provides protection levels on the order of $2 \mathrm{~m}$ at almost all times where DGPS alone only rarely has observation redundancy and often exhibits estimated accuracies worse than $200 \mathrm{~m}$. Reliability is further improved when the vehicle azimuth is assumed to be known a priori.
\end{abstract}

\section{Introduction}

While single point GPS positioning may be sufficient for user navigation and guidance on most highways, other applications require a more precise intervehicle relative position. This paper assesses the utility of integrating between-vehicle differential GPS with direct measurements of intervehicle range using ultrawideband ranging (UWB) radios and direct observation of intervehicle bearing.

Relative positioning between vehicles, or vehicle-tovehicle (V2V) navigation, using GPS has been a topic of interest for at least a decade [1]. With more and more vehicles equipped with GPS receivers, the establishment of ad hoc networks of vehicles exchanging position information becomes possible and enables many vehicle navigation applications that depend on relative positioning. Some examples include slow or stopped vehicle ahead, vehicle in blind-spot warnings, or smart cruise control coordination for vehicles travelling in platoons on highways [2].
While relative GPS can provide V2V navigation in many environments, in urban canyons and under dense foliage GPS alone may not be able to provide a reliable solution estimate [3], and information from other vehicle sensors will be required [4]. In this light, UWB is a relatively new technology, having only been FCC approved in 2002. UWB ranging radios operate by measuring a two-way time of flight with $\mathrm{cm}$ level precision and multipath resistance $[5,6]$ using a series of nanosecond length pulses. Previous work has demonstrated the advantages of augmenting GPS with UWB ranges for static users and for slowly moving users making observations to stationary UWB radios located over known points $[7,8]$.

UWB is a promising technology for direct ranging between vehicles. This ability would be even more valuable if the angle-of-arrival (or bearing from one vehicle to another as measured in the body frame of the first vehicle) could also be measured. Being able to measure both of the distances and bearings to a network of nearby vehicles would result in 
good overall network geometry and the possibility of reliable relative navigation even in the total absence of GPS signals. While systems to measure bearing using UWB ranging signals have been developed [9], there are other possible ways to measure bearing including using digital cameras, vehicle mounted radars, and so forth.

The purpose of this paper is to assess the usefulness of UWB ranging and bearing measurements to augment GPS as a means of determining the relative positions of a network of moving vehicles. Specifically this paper analyzes, through covariance analysis, the effect of adding direct between-vehicle UWB range and bearing measurements to networks of vehicles separated by less than $300 \mathrm{~m}$ that are using code (pseudorange) DGPS methods to establish their relative horizontal positions. It is noted that the use of carrier phase data would theoretically provide considerably higher accuracy than using pseudoranges (e.g., $[1,10]$ ), but this is not considered herein for several reasons. First, consideration is given to environments with limited satellite visibility where ambiguity resolution is already known to be extremely difficult, if not impossible. Second, even if carrier phase were considered, it is very difficult to accurately consider all possible error effects thus resulting in overly optimistic results [11]. Third, automotive grade antennas are typically roofmounted and designed to handle several frequency bands (e.g., GNSS, mobile phone, XM, AM, FM, etc.) and do not usually provide any multipath mitigation, meaning that carrier phase data quality may not even be useful. Fourth, practically speaking, V2V positioning systems are still in their relative infancy, and a logical first step would be to use a pseudorange-only approach. Finally, many consumer GPS receivers do not currently output carrier phase data, meaning that any insights from a carrier phase analysis would be largely unrealizable in the near future.

The remainder of this paper is organized as follows: Section 2 describes how GPS pseudoranges, UWB ranges, and bearing observations are combined in a relative navigation solution. In Section 3 this approach is evaluated by means of a covariance simulation study conducted in a simulated urban canyon environment with up to nine vehicles participating in a relative navigation network. In Section 4 the results of this simulation are then analysed in terms of the effects of different qualities of observations, network geometries, numbers of participating vehicles, and filtering assumptions on the achievable accuracy and reliability of the relative navigation solution.

\section{Measurement Models and Integration Strategies}

This section presents the mathematical models for the various measurements used. The integration algorithm is then described.

2.1. GPS Models. The mathematical models for GPS positioning are well known [12]. In this work, the double difference (DD) formulation has been adopted in order to simplify the analysis by eliminating the need to estimate a GPS receiver clock offset. The DD pseudorange measurement between the $a$ th and $b$ th receivers and the $i$ th and $j$ th satellites is written as

$$
\nabla \Delta P_{a b}^{i j}=\nabla \Delta \rho_{a b}^{i j}+\nabla \Delta \varepsilon_{a b}^{P}
$$

where $\nabla \Delta$ is the DD operator such that $\nabla \Delta(\bullet)_{a b}^{i}=(\bullet)_{a}^{i}-$ $(\bullet)_{b}^{i}-(\bullet)_{a}^{j}+(\bullet)_{b}^{j}, P$ is the pseudorange measurement, $\rho$ is the geometric range between the satellite and the receiving antenna, and $\varepsilon^{P}$ represents the combination of all pseudorange errors. The undifferenced geometric range can be written mathematically as

$$
\rho_{a}^{i}=\left\|\vec{r}^{i}-\vec{r}_{a}\right\|
$$

where $\vec{r}^{i}$ is the position vector of the $i$ th satellite and $\vec{r}_{a}$ is the position vector of the $a$ th receiver.

The largest spatially correlated differential error source is the ionosphere, which has a typical spatial correlation of about 3 ppm [13]. However, for the intervehicle distances considered in this work, namely, less than $300 \mathrm{~m}$, this contributes to only $0.9 \mathrm{~mm}$ of error, which is well below the pseudorange measurement noise. As such, all spatially correlated errors are hereafter assumed to be zero and the only relevant errors are assumed to be multipath, and noise.

Equation (1) is linearized about the current position estimates to give

$$
\begin{aligned}
\nabla \Delta P_{a b}^{i} \approx & \nabla \Delta \hat{\rho}_{a b}^{i}+\left.\frac{\partial \nabla \Delta P_{a}^{i}}{\partial \vec{r}_{a}}\right|_{\vec{r}_{a}=\hat{\vec{r}}_{a}} \delta \vec{r}_{a}-\left.\frac{\partial \nabla \Delta P_{b}^{i}}{\partial \vec{r}_{b}}\right|_{\vec{r}_{b}=\hat{\vec{r}}_{b}} \delta \vec{r}_{b} \\
& +\nabla \Delta \varepsilon_{a b}^{P}, \\
\nabla \Delta P_{a b}^{i}- & \nabla \Delta \hat{\rho}_{a b}^{i}=H_{a} \delta \vec{r}_{a}-H_{b} \delta \vec{r}_{b}+\nabla \Delta \varepsilon_{a b}^{P},
\end{aligned}
$$

where an "over-hat" (i.e., $\widehat{\bullet})$ represents the current best estimate of a parameter (with $\hat{\rho}_{a b}^{i}$ being computed from $\widehat{\vec{r}}_{a}$ and $\widehat{\vec{r}}_{b}$ ), $\delta$ indicates an error in the following parameter, and $H$ is the Jacobian matrix of the observations at the subscripted receiver. The Jacobian can be shown to consist of unit vectors pointing from the satellite to the receiver [14]. Because of the large distance between the receivers and the satellites $(20,000 \mathrm{~km}$ or more) and the relatively short distance between receivers (less than $300 \mathrm{~m}$ ), the unit vectors for the two receivers can be reasonably assumed to be the same. As such, and denoting $H=H_{a} \approx H_{b}$, (3) is simplified to

$$
\begin{aligned}
\nabla \Delta P_{a b}^{i}-\nabla \Delta \hat{\rho}_{a b}^{i} & \approx H\left[\delta \vec{r}_{a}-\delta \vec{r}_{b}\right]+\nabla \Delta \varepsilon_{a b}^{P} \\
& =H \delta \Delta \vec{r}_{a b}+\nabla \Delta \varepsilon_{a b}^{P},
\end{aligned}
$$

where $\Delta \vec{r}_{a b}$ is the relative position of receivers $a$ and $b$. The importance of (4) is that it shows that DD pseudorange measurements can be used to directly observe relative positions. Since this work is ultimately interested in estimating relative positions of vehicles, (4) is the fundamental measurement model used herein. 


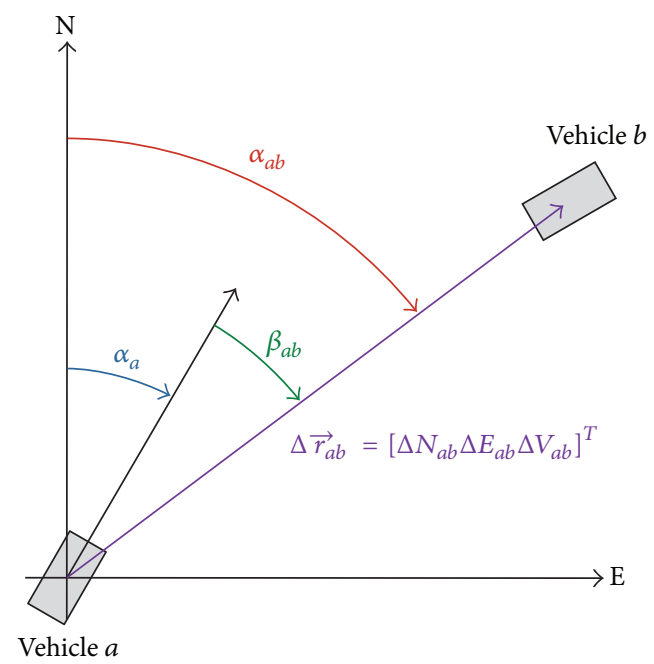

FIGURE 1: Graphical representation of a bearing measurement.

2.2. UWB Range Model. The second measurement considered in this work is the UWB range measurement, $P_{a b}^{\mathrm{UWB}}$, between radios $a$ and $b$. The measurement model is given by

$$
P_{a b}^{\mathrm{UWB}}=\rho_{a b}+\varepsilon_{a b}^{\mathrm{UWB}},
$$

where $\rho_{a b}$ is the geometric distance between the two radios and $\varepsilon_{a b}^{\mathrm{UWB}}$ are the UWB measurement errors. It is noted that there is no receiver clock error and that the measurement errors are different than in the GPS case and instead include bias and scale factor errors, multipath and noise [15]. In this paper the bias and scale factor effects have been ignored as they are on the order of code DGPS position errors, but as shown in [16] they do have to be taken into account in order to obtain a UWB-aided carrier phase solution. Linearizing (5) gives

$$
P_{a b}^{\mathrm{UWB}}-\hat{\rho}_{a b}^{\mathrm{UWB}}=H\left[\delta \Delta \vec{r}_{a b}\right]+\varepsilon_{a b}^{\mathrm{UWB}} .
$$

Equation (6) shows that the UWB data directly observes the relative position of the two receivers.

2.3. Bearing Model. The final measurement considered herein is the bearing measurement. Bearing is an angular measure of the horizontal direction of another vehicle relative to the forward direction of the vehicle making the measurement. The concept is shown graphically in Figure 1, where $\beta_{a b}$ is the bearing measurement from vehicle $a$ to $b$. Also shown, are the azimuth of vehicle $a$, denoted by $\alpha_{a}$, and the azimuth of the relative position vector $\left(\Delta \vec{r}_{a b}\right)$, denoted by $\alpha_{a b}$.

From Figure 1, the bearing measurement can be written mathematically as

$$
\beta_{a b}=\alpha_{a b}-\alpha_{a}
$$

However, the azimuth of the relative position vector can be written as

$$
\alpha_{a b}=\tan ^{-1}\left(\frac{\Delta E_{a b}}{\Delta N_{a b}}\right),
$$

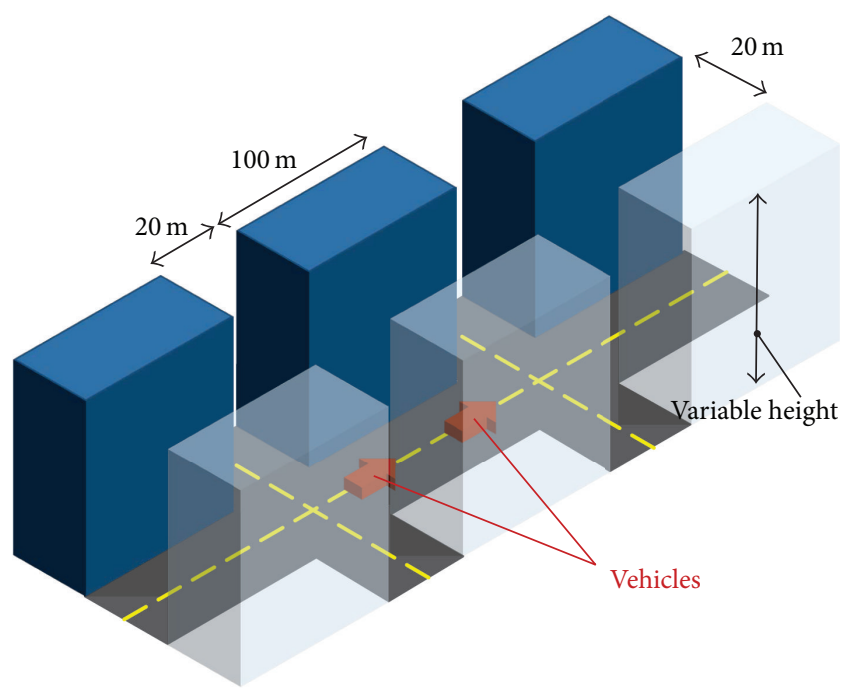

FIGURE 2: Diagram of the simulated urban canyon environment.

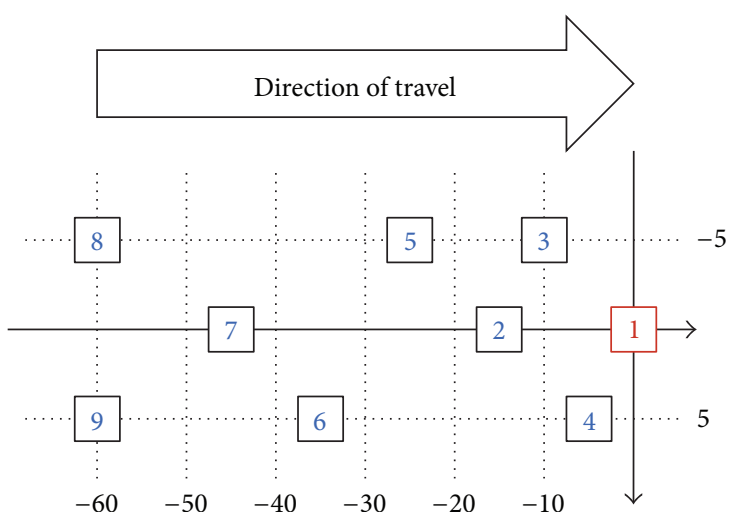

FIGURE 3: Relative locations of vehicles driving in simulated urban canyon environment.

Where $\Delta N_{a b}, \Delta E_{a b}$, and $\Delta V_{a b}$ (shown in Figure 1) are the north, east, and vertical components of the relative vector in the local level frame. Substituting this result into (7) gives the final equation for the bearing measurement:

$$
\beta_{a b}=\tan ^{-1}\left(\frac{\Delta E_{a b}}{\Delta N_{a b}}\right)-\alpha_{a} .
$$

Linearizing (9) and adding measurement noise, $\varepsilon_{a b}^{\beta}$, gives

$$
\beta_{a b}-\widehat{\beta}_{a b}=H_{\beta}\left[\delta \Delta \vec{r}_{a b}\right]-\delta \alpha_{a}+\varepsilon_{a b}^{\beta}
$$

It is important to note that to use the bearing measurement one needs to know-or have an estimate of - the azimuth of the vehicle making the measurement (vehicle $a$ in the above development). This is needed to relate the bearing measurement, which is made in the frame of the vehicle making the measurement, to a globally referenced frame (in this case, the local level frame). In turn, this leads to two separate implementation options. In the first case, the azimuth of the vehicle making the bearing measurement 

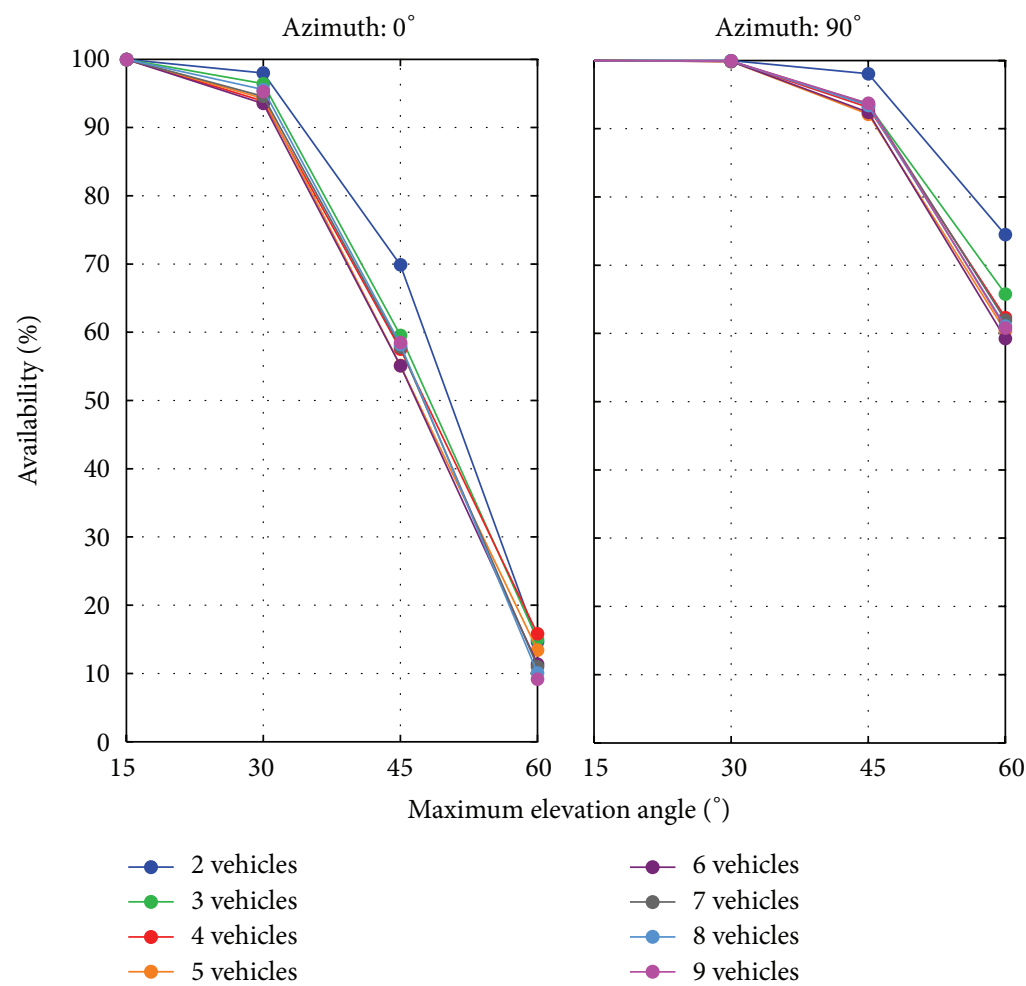

FIGURE 4: GPS-only availability for different network sizes as a function of maximum elevation angle for road azimuths of $0^{\circ}$ and $90^{\circ}$.

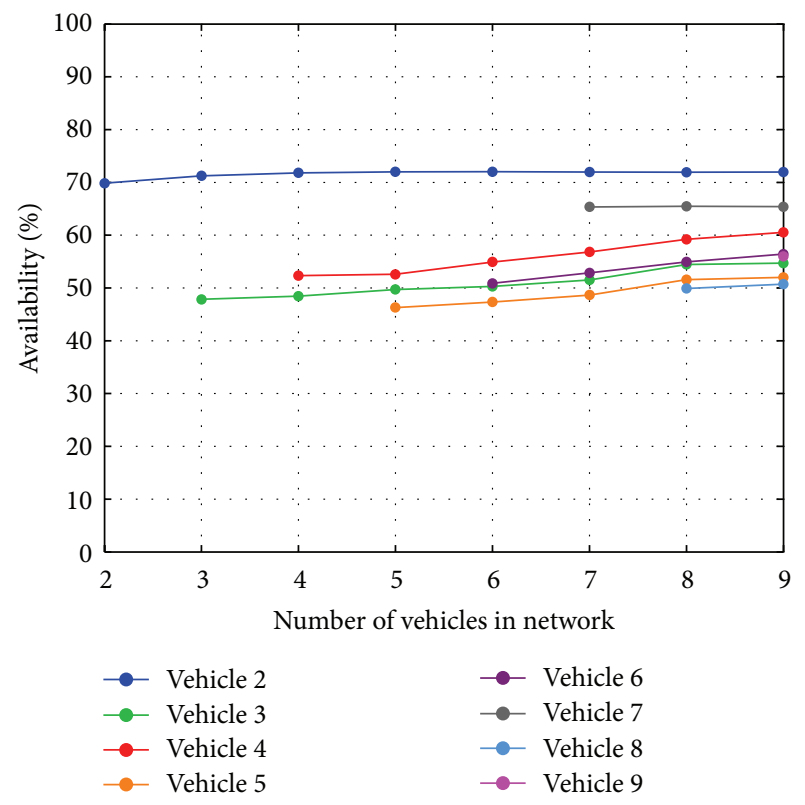

Figure 5: Solution availability of each vehicle as a function of the number of vehicles in the network for a $0^{\circ}$ road azimuth and a $45^{\circ}$ maximum elevation angle.

is assumed to be known, for example, from an onboard navigation system or other sensors. While a GPS-based attitude system could also be used to obtain the azimuth, given the complexities involved in developing and deploying a system with multiple antennas and receivers, adding a second GPS receiver solely for attitude information is not a likely scenario in the short term. In this case, the $\delta \alpha$ term in (10) is zero (i.e., no error in the estimated value). The second case assumes the azimuth of the vehicle making the bearing measurement is unknown and must, therefore, be estimated along with the relative position states. The former case is preferred, since it removes one unknown for every bearingmeasuring vehicle in the network. Both cases are considered in this work.

2.4. Integration Filter. As mentioned in the previous section, the primary GPS measurements used are the DD pseudoranges. Correspondingly, it is apparent from (4) that by using these measurements, the absolute position of the receivers cannot be determined. To account for this, we herein refer to a "lead vehicle" whose position is assumed to be known. The absolute positioning accuracy of the lead vehicle is not very stringent. Specifically, with reference to Tang [17] the effect of reference station (lead vehicle) position error on the baseline solution is closely approximated by

$$
\|\delta \Delta \vec{r}\| \approx \frac{10^{-9}\left\|\delta \vec{r}_{\text {base }}\right\|}{\|\Delta \vec{r}\|},
$$

where $\delta \vec{r}_{\text {base }}$ is the error in base station position and $\Delta \vec{r}$ is the baseline vector. For the application at hand, even if the lead vehicle has an absolute positioning error as large as $3 \mathrm{~km}$, the resulting error on the (maximum) intervehicle distance of $300 \mathrm{~m}$ will be on the order of $10^{-8} \mathrm{~m}$, which is negligible. It is 

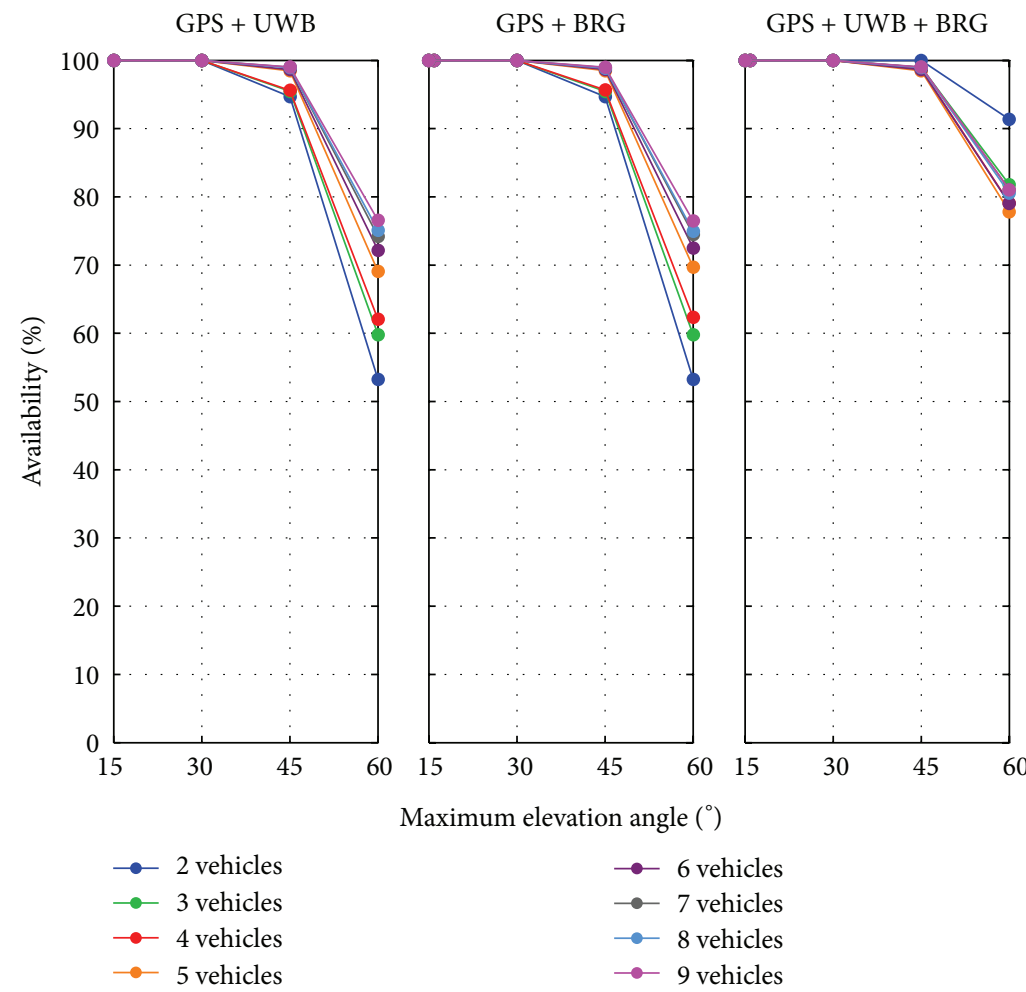

FIGURE 6: Solution availability for the $0^{\circ}$ road azimuth for GPS + UWB, GPS + bearing, and GPS + UWB + bearing as a function of maximum elevation angle and number of vehicles.
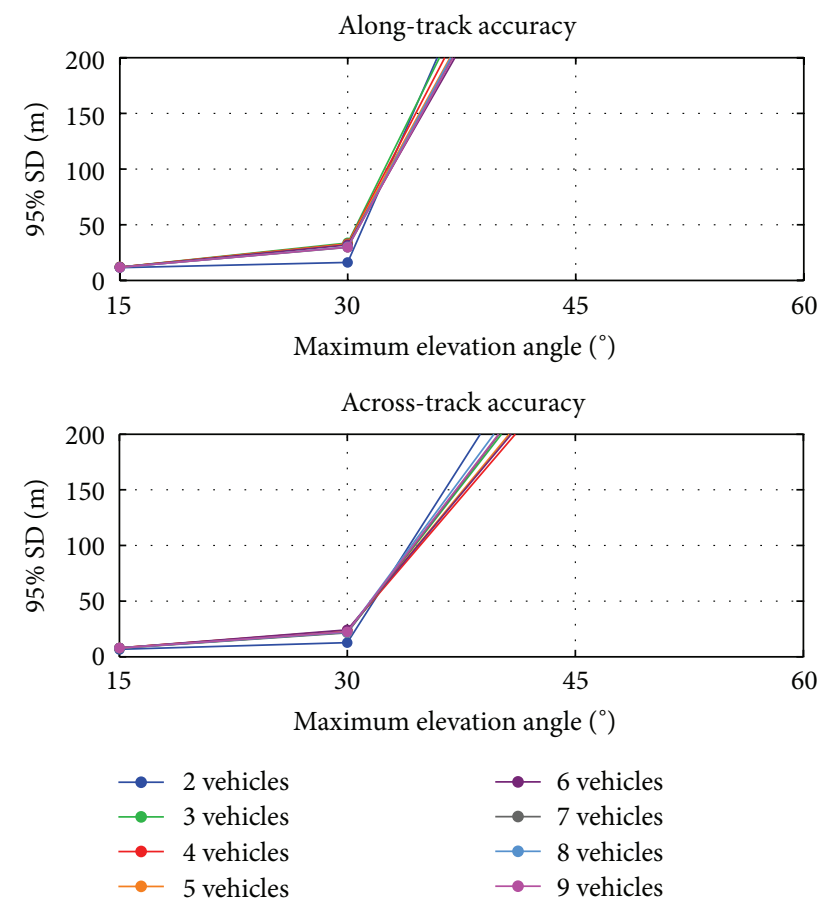

FIGURE 7: 95th percentile along-track and across-track accuracy using GPS as a function of the number of vehicles in the network and the maximum elevation angle for a road azimuth of $0^{\circ}$. safe to assume the position of the lead vehicle can be easily determined to this level of accuracy using standalone GPS data and/or other data that may be available.

The covariance analysis presented in this work focuses primarily on the use of least-squares (LS). This is done because the relative benefit of the UWB and bearing data on the relative position solution can be more easily assessed in the absence of filtering, which can significantly affect performance. That said, for completeness, comparisons between least-squares and Kalman filtering (KF) are presented at the end of the paper. Details of least-squares and Kalman filter algorithms are omitted here, and interested readers are referred to the many publications on these subjects (e.g., [1820]).

The elements of the estimated state vector vary according to what type of measurements are being used. In all cases the state vector includes a $3 \times 1$ local level position vector for every non-lead vehicle (this vector is a relative position to the lead vehicle). As necessary, the azimuth of all bearing-measuring vehicles is also included.

Only pseudorange data is used from the GPS receiver in all cases. The inclusion of UWB and/or bearing measurements is used to assess their relative benefit to the GPSonly solution. For simplicity, GPS pseudorange measurement accuracy is assumed to be independent of satellite elevation.

For vehicle networks consisting of more than two vehicles, only linearly independent observations were used to 
avoid a singular solution with least-squares. The algorithm described by Saalfeld [21] was used to select independent measurements. Finally, the covariance matrix of the double difference pseudoranges was fully populated to account for correlation between measurements arising from the betweensatellite differencing process.

\section{Covariance Analysis Description}

This section discusses the covariance simulations conducted and the metrics used to assess the performance of the different scenarios.

\subsection{Simulation Description}

3.1.1. Simulated Environment. The simulated environment models an urban canyon with all vehicles moving along a road with a constant azimuth and with buildings on either side. All buildings have the same dimension (specifiable) and are assumed to each occupy an entire city block. Buildings continue to infinity in the forward and reverse directions. A diagram of the urban canyon environment used in this work is shown in Figure 2. Note that in this case the building geometry repeats every $120 \mathrm{~m}$.

To assess the impact of the size of the vehicle network on performance, up to nine vehicles were simulated traveling in the same direction. The relative position of the vehicles is shown in Figure 3. Vehicles are added to the network according to the numbers shown. For example, the threevehicle network consists of vehicles numbered one to three.

Throughout the simulation, the vehicles maintain the same geographic position (i.e., latitude and longitude) and the buildings are simulated to move past the vehicles. This approach is preferable to having the vehicle positions change over time because in the latter case the vehicle positions would differ between simulation runs depending on the azimuth of the road. In turn, the simulated vehicles would observe satellite geometry that is not solely affected by the azimuth of the road (and thus the geometry of the obstructions) but also contains effects due to different geographic locations. It was felt that this would further complicates the analysis. With this in mind, all simulation results are for Calgary, AB, Canada. Similar results were obtained for other North American cities but are not included in the interest of space.

The buildings were simulated to move at a rate of $0.2 \mathrm{~m} / \mathrm{s}$. This allows the building geometry to repeat every ten minutes (i.e., $120 \mathrm{~m} / 0.2 \mathrm{~m} / \mathrm{s}=600 \mathrm{~s}$ ), over which time the satellite geometry (in the absence of obstructions) does not change very much. In terms of the data analysis, the assumption is that the satellite geometry is approximately constant over ten minutes and so any variations in performance within that time interval are attributed mostly to the location of the vehicles within the urban canyon. Furthermore, the slow motion of the buildings means that the location of the vehicles within the urban canyon is sampled with sufficiently high resolution. In other words, the simulation should capture a representative range of satellite geometries resulting from the combination of satellite motions and locations of the vehicles within the urban canyon.

The height of the building and the azimuth of the road were left as specifiable parameters in the simulations. The road azimuth was selected as $0^{\circ}$ or $90^{\circ}$. The heights of the buildings were computed based on the maximum elevation angle they would produce for a vehicle located in the centre of the road. The maximum elevation angles were varied from $15^{\circ}$ to $60^{\circ}$ in $15^{\circ}$ increments. An elevation mask of $10^{\circ}$ was used for the forward and reverse directions (since there are no simulated buildings in those directions).

The simulations were run over 24 hours with measurements every $5 \mathrm{~s}(0.2 \mathrm{~Hz})$. The GPS almanac used was taken from GPS week 1587 and consisted of 30 healthy satellites. It is noted that most GPS receivers allow measurements at a rate of $1 \mathrm{~Hz}$ or higher, which is higher than the rate of $0.2 \mathrm{~Hz}$ used in the simulations. Recall that the main objective of the covariance simulation is to assess the benefit of including UWB and bearing data, particularly when GPS availability is limited. As such, the "absolute" simulated data rate itself was not considered a critical parameter in the simulation. Instead, the results are most affected by GPS satellite geometry changes due to satellite motion and the local signal masking environment. With this in mind, the use of a $0.2 \mathrm{~Hz}$ measurement rate will still give a very high "resolution" in terms of satellite motion. As mentioned above, the effect of local signal masking is addressed by selecting a suitably appropriate velocity for the buildings.

3.1.2. Simulated Measurements. The GPS measurement simulation is typical of similar studies. The satellite geometry is evaluated and satellites that are in view (above the elevation mask and not blocked by the simulated urban canyon) are assumed to be available for positioning.

UWB ranges are assumed to be available between all vehicles in the network, but each distance is only observed one way (e.g., from vehicle 1 to 2 but not the other way). If measurements made in the other direction were included, the net effect would be the same as the one-way case but with the measurement accuracy reduced by $\sqrt{2}$.

The bearing sensor is simulated as a device that is capable of measuring the angle between the "forward" axis of the vehicle body frame and the line of sight to the other vehicle. Bearing measurements are simulated from each vehicle to all the vehicles "in front" of it. In this context, "in front" refers to the vehicle number, not necessarily their relative position. For example, vehicle 4 measures a bearing to vehicle 3 , even though it is behind it, and similarly, vehicle 3 does not observe the bearing to vehicle 4 . This was done to limit the number of observations and also to ensure that each nonlead vehicle is making at least one bearing observation. This enables each nonlead vehicle to have an observable azimuth in the case where the azimuth is assumed to be unknown, thus simplifying software development.

The measurements were assigned the (undifferenced) measurement accuracy shown in Table 1. The GPS measurement accuracy was chosen to incorporate the effects of code multipath. It is acknowledged, however, that since multipath 
TABLE 1: Standard measurement accuracies used for covariance simulations.

\begin{tabular}{lc}
\hline Measurement & Accuracy $(1 \sigma)$ \\
\hline GPS pseudorange & $5 \mathrm{~m}$ \\
UWB range & $0.5 \mathrm{~m}$ \\
Bearing & $0.5^{\circ}$ \\
\hline
\end{tabular}

is highly variable, this value may be a bit small. Nevertheless, it serves the purpose of assessing the relative benefit of the other sensors on the overall solution.

The UWB error of 0.5 metres is based on previous tests conducted with UWB radios under the assumption that nothing is being done to estimate or calibrate the various systematic errors present in current UWB radios [15].

The bearing sensor accuracy was selected to represent what is expected on commercial vehicles in the near future [22].

3.2. Performance Metrics. The performance metrics used herein include availability, accuracy, and reliability.

Availability refers to the percentage of the time a system is able to provide the user with navigation solutions [23]. In the context of GPS, availability relates to the number of satellites available to the user. This is the definition of availability that was used in this paper. Specifically, if a solution can be computed using a least-squares approach, the solution is considered "available."

The accuracy of the solution is assessed using the estimated uncertainties in the horizontal directions, as obtained from the state covariance matrix of the least-squares or Kalman filter solution. These uncertainties are rotated from the local level frame into the frame defined by the forward direction of the vehicle (using the known road azimuth) in order to show the along- and across-track uncertainties. The motivation for this is that along- and across-track uncertainties are generally more useful for making vehiclecentric decisions such as "is the vehicle ahead in the same lane as I am?" or "is there a vehicle in the lane next to me?"

Finally, reliability refers to the ability to detect blunders in the measurements and to estimate the effects of undetected blunders on the navigation solution. Reliability is further divided into internal and external reliability, both of which are based on assumed probabilities for rejecting a good observation (5\% in this work) and accepting a biased observation (10\% in this work). Internal reliability refers to the smallest measurement bias-often called the marginal detectable bias (MDB) - that can occur and still be detected. In contrast, external reliability is the worst case error in the estimated parameters that result from an MDB occurring on a single measurement at a given epoch and is typically called a protection level (PL). Similar to the accuracy assessment, the protection level is computed in the along- and across-track directions for convenience.

Details regarding how to compute reliability parameters can be found, for example, in Leick [14] and FRP [23] for the least-squares case and in Teunissen and Salzmann [24] and Koch [20] for the Kalman filter case, and are thus not
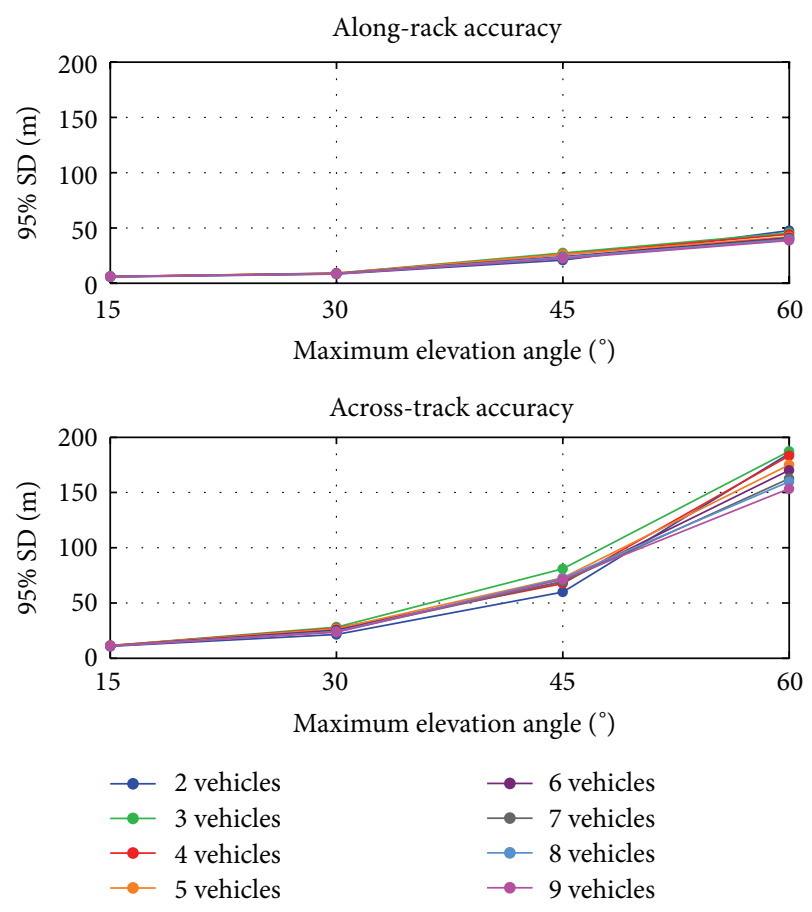

FIGURE 8: 95th percentile along-track and across-track accuracy using GPS as a function of the number of vehicles in the network and the maximum elevation angle for a road azimuth of $90^{\circ}$.

repeated here. However, it is noted that there are cases where measurement redundancy is very low such that the MDB can be larger than the largest possible error that can occur in the particular sensor. For example, in a scenario where the GPS position accuracy is very poor in the along-track direction and a UWB range is used, the solution may be depending entirely on the UWB range to control the alongtrack component. This means that the redundancy of the UWB observation is almost zero and the remaining GPS observations would have little to no ability to detect a blunder in the UWB range. As a result, the computed MDB for the UWB range may be $100 \mathrm{~s}$ of metres when the radios themselves are only capable of measuring $300 \mathrm{~m}$ and almost always have errors of a metre or less. Similarly, if there is a bearing observation that is providing the only observation in the across-track direction, the MDB on that measurement may be larger than physically reasonable, for example, greater than $180^{\circ}$ (implying the sensor is no longer facing forward). In both cases, MDBs that are larger than physically possible will map to protection levels that are unrealistically large. With this in mind, the MDB for a UWB measurement has been limited to $300 \mathrm{~m}$, and for a bearing observation it has been limited to $\pm 180^{\circ}$.

\section{Analysis of Results}

As mentioned earlier, most of the analysis focuses on the least-squares results, as this allows for the most obvious assessment of the benefit of UWB and bearing measurements. Kalman filtering results are discussed only briefly towards the 

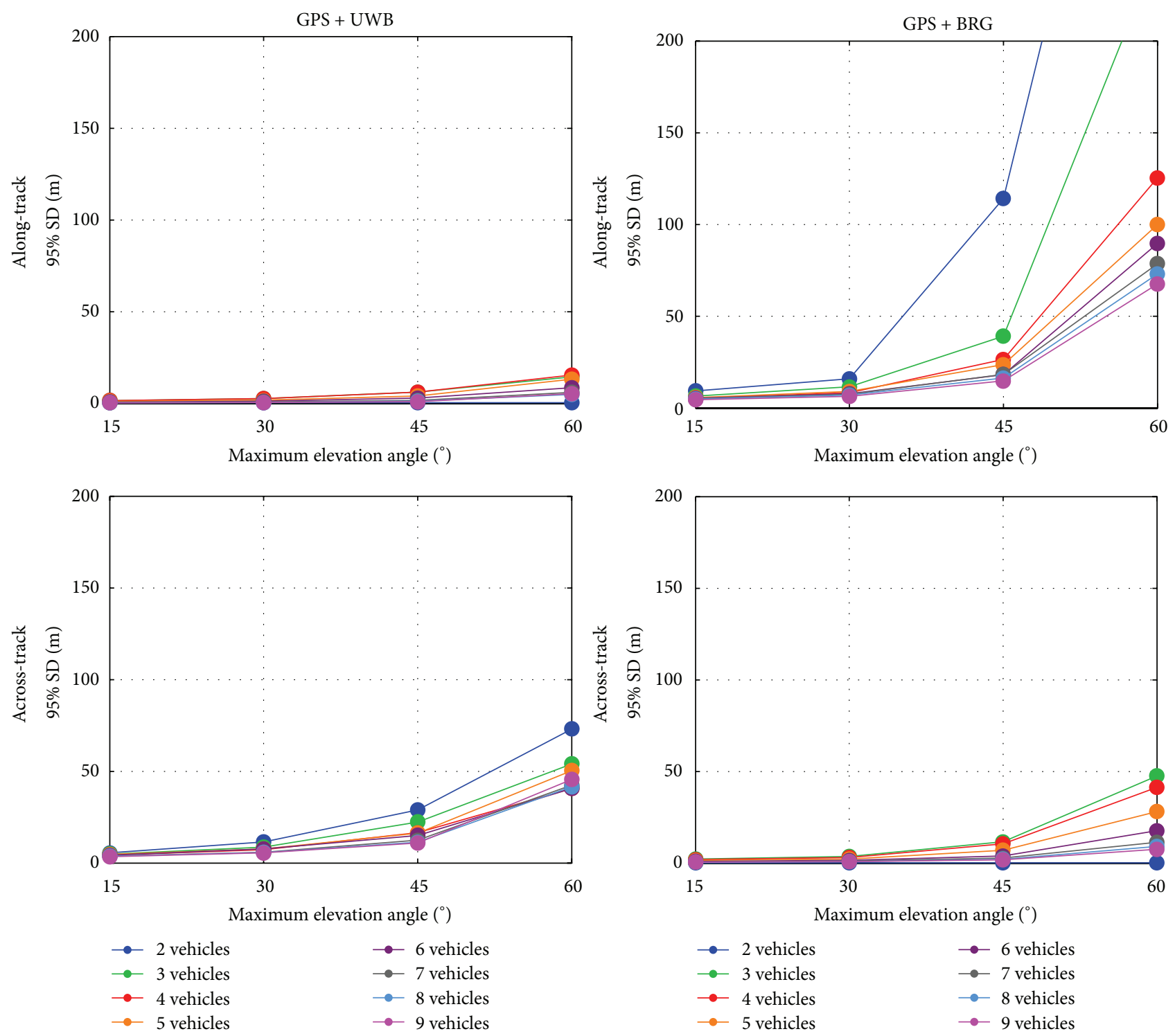

FIGURE 9: 95th percentile along-track and across-track accuracy using GPS + UWB (left) and GPS + bearing (right) as a function of maximum elevation angle for road azimuths of $0^{\circ}$.

end to give an idea of the possible improvements that could be achieved through modelling of vehicle dynamics and use of observations from multiple epochs.

Also, given the number of parameters at play, it is impossible to show all results in a concise manner. As such, only the most interesting findings are included explicitly. Whenever possible, however, comments regarding other findings are described in the text.

Before continuing, unless stated otherwise, inclusion of bearing measurements assumes the azimuth of the vehicle making the measurements is known.

4.1. Availability. Figure 4 shows the solution availability (percentage of the time that any given vehicle in the network has a solution) for the GPS-only case as a function of urban canyon elevation angle and the number of vehicles in the network for the two different road azimuth configurations simulated. As vehicles are added, they will either have or not have sufficient observations to position themselves with respect to the other vehicles, and each vehicle is included in computing the availability. As a result the addition of vehicles tends to lower the solution availability. For example, for the $0^{\circ}$ road azimuth (left plot) with a 45-degree maximum elevation angle, two vehicles have a relative position solution $70 \%$ of the time. With three vehicles, the two baselines (three vehicles) are available $60 \%$ of the time for the same elevation angle (which is what is plotted) even if the first baseline (first two vehicles) are available $70 \%$ of the time individually.

Another important point to note in this figure is that the $0^{\circ}$ road azimuth is considerably worse than for $90^{\circ}$. This is because the GPS satellite orbit inclination angle of $55^{\circ}$ results 

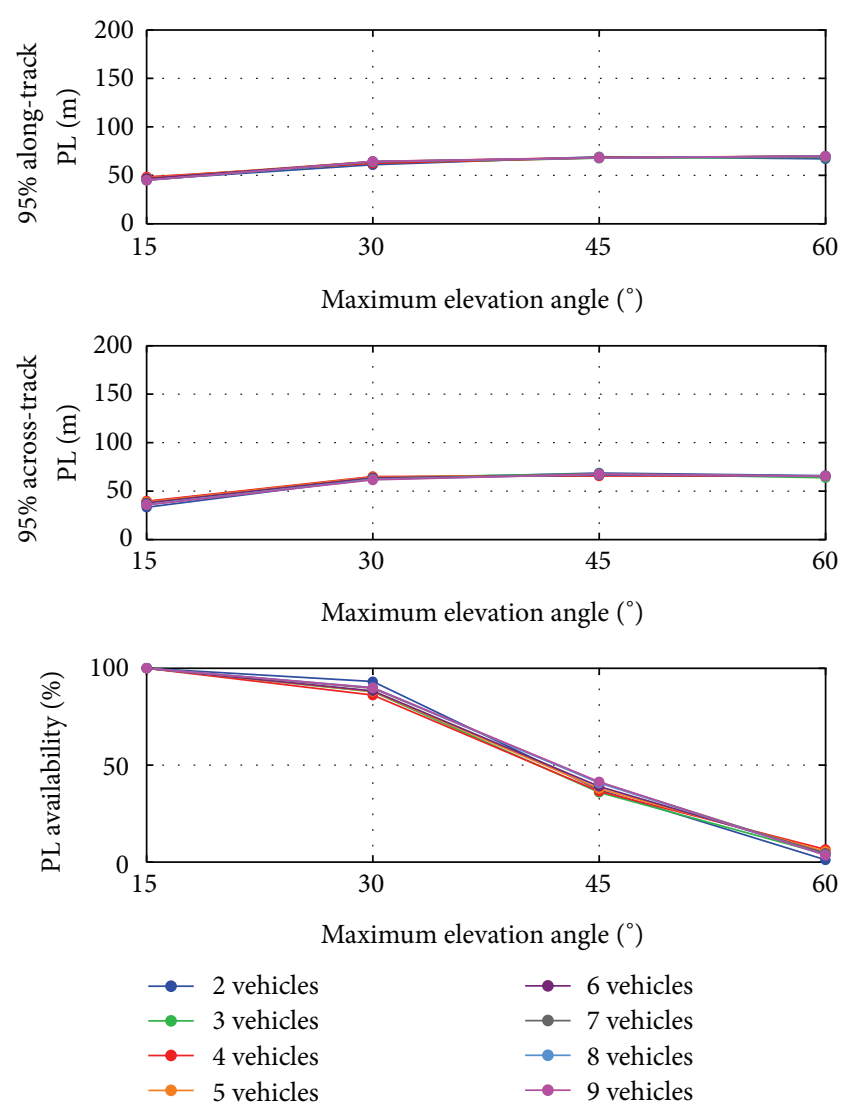

FIGURE 10: 95th percentile along-track and across-track protection levels and protection level availability using GPS as a function of maximum elevation angle for a road azimuth of $0^{\circ}$.

in GPS satellites rarely being visible directly to the north for users in Calgary (simulated location). In a north-south urban canyon, there are effectively no satellites directly to the north, few to the south, and the satellites on either side (east or west of the user) are obstructed by buildings. In contrast, when the canyon is oriented east-west, there is good along-track visibility (satellites to the east and west of the user) while the canyon blocks the north and south, where there are fewer satellites to begin with. The effect is particularly visible for the steepest canyon $\left(60^{\circ}\right)$ where there is very little availability for the $0^{\circ}$ azimuth case and over $60 \%$ in all cases for the $90^{\circ}$ azimuth canyon.

If other satellite-based navigation systems were incorporated into the solution (e.g., GLONASS, Galileo, Beidou/Compass, etc.), the availability would improve, perhaps significantly. This is not considered here in order to keep the analysis more tractable and because currently, GPS is the only system available on most vehicles-although this is changing. In addition, if the availability benefits of adding other systems can be quantified, the results in this paper can still be used to infer the relative benefits of UWB and bearing sensors. For example, consider the left plot in Figure 4 (i.e., $0^{\circ}$ azimuth) with a maximum elevation angle of $60^{\circ}$. If adding GLONASS improves the availability to around $60 \%$, this is similar to the results obtained with an elevation mask of $45^{\circ}$ and the result presented below can still be used to approximate the improvements brought from other sensors.

To better illustrate the solution availability differently, Figure 5 shows the percentage availability of each vehicle as a function of the number of vehicles in the network. Note that, for example, the first two vehicles' availability improves as more vehicles are added, but that these new vehicles have poorer availability than the first two. This is because all but one of the additional vehicles are located closer to one of the two walls of the urban canyon and hence have an even poorer view of the sky than the first two. Vehicle 7 is also in the middle of the road and has the next best availability after the first two.

Having shown the GPS-only availability, Figure 6 shows the availability when adding UWB ranges (GPS + UWB), bearing measurements (GPS + BRG) and both UWB and bearing. The addition of UWB measurements or bearing measurements has a very similar effect because in both cases a similar number of observations are being added. As such, if in the GPS-only case there is a vehicle that is short, one observation, and is listed as "not available," an additional observation will move that vehicle to the "available" category regardless of whether the observation is a range or a bearing. Note that this is only true in the case that the vehicle making the bearing observation has a known azimuth, which is assumed here.

Also, with both observation types added there is effectively $100 \%$ availability except for the case with the most severe elevation mask. This is not surprising since a range and bearing between two vehicles are sufficient to determine the relative horizontal position (even if GPS were not available at all). This is equivalent to having a radar-type system that can provide bearing and range from the sensor equipped vehicle to the "target" vehicle. For the $60^{\circ}$ mask, there are several epochs with less than $100 \%$ availability because there are no GPS satellites to measure the vertical channel.

Finally, unlike the GPS-only case, as more vehicles are added the availability tends to improve. This is because each vehicle that is added now also results in several additional UWB and bearing measurements (recall that each vehicle is ranging to all others and each new vehicle added has bearing measured to each existing vehicle in the network).

4.2. Accuracy. Since the estimated accuracy will vary over time due to changes satellite geometry and also because of a vehicle's location relative to the simulated buildings, it is impractical to show all results here. Instead, the 95th percentile uncertainties (standard deviations) are plotted. Furthermore, these percentile values are computed across all vehicles in the network.

Figures 7 and 8 show 95th percentile values of the estimated along- and across-track position error as a function of maximum elevation angle and the number of vehicles in the network for $0^{\circ}$ and $90^{\circ}$ road azimuths, respectively. These results illustrate two main findings. First, as suggested by the availability analysis above, these results show that the $0^{\circ}$ road azimuth case is poorer than the $90^{\circ}$ road azimuth 
GPS + UWB
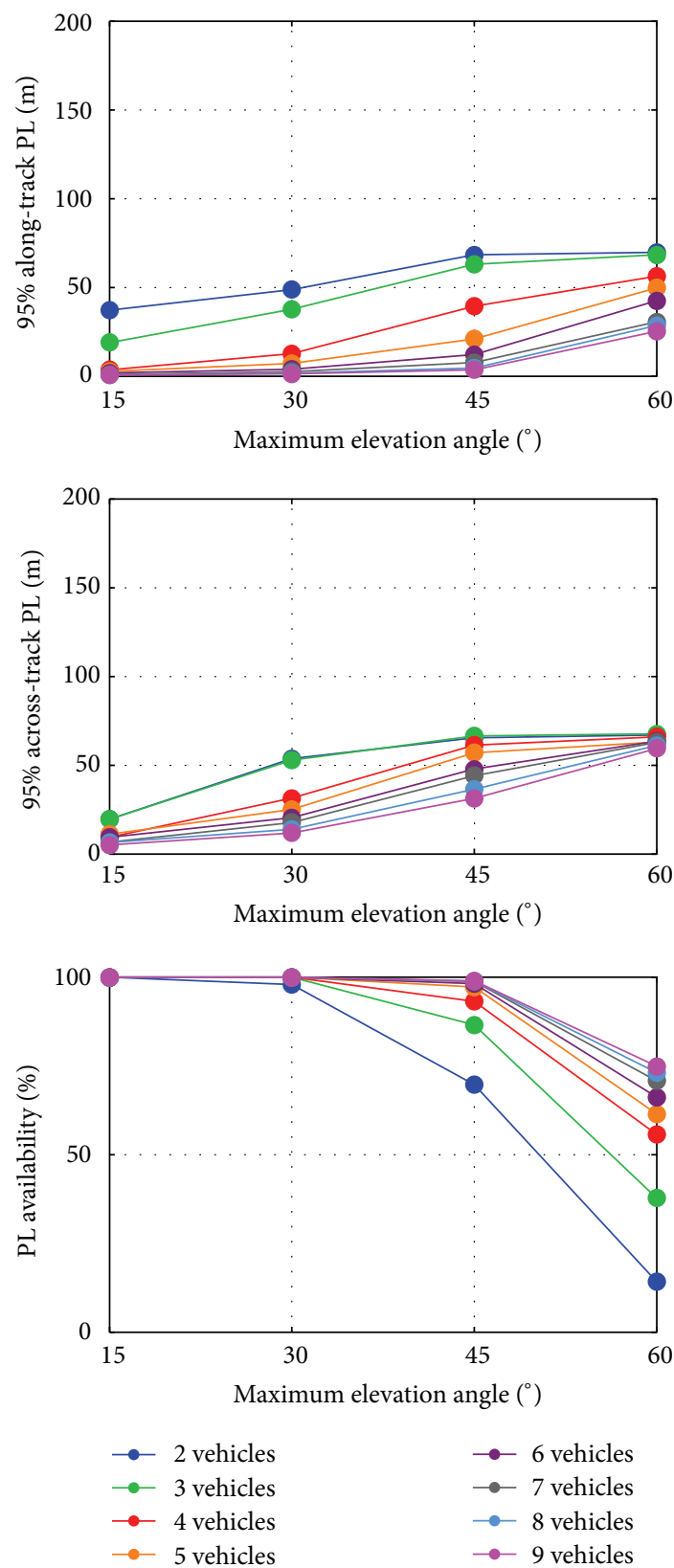

GPS + BRG
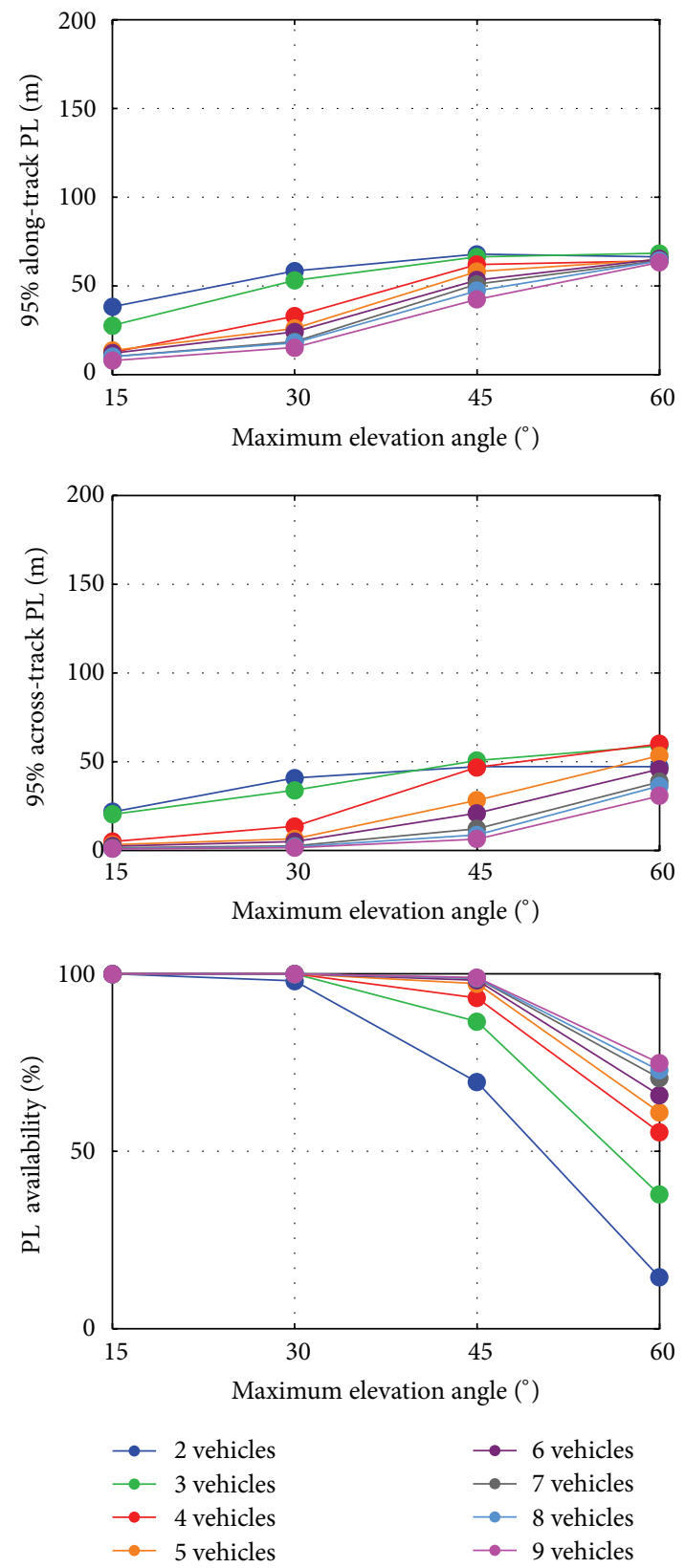

FIGURE 11: 95th percentile along-track and across-track protection levels and protection level availability using GPS + UWB (left) and GPS + bearing (right) as a function of maximum elevation angle for a road azimuth of $0^{\circ}$.

case. Second, the along-track component is always better than the across-track component. Both of these results are due to satellite geometry and the fact that in an urban canyon the satellites on either side of the vehicles are obscured making across-track relative navigation more problematic than along-track. This suggests that GPS alone, under low signal masking conditions may be sufficient for certain applications requiring along-track relative positioning (e.g., warning of a slower vehicle ahead) but may be less capable of providing across-track information, for example, lane control.
The other point to note from Figures 7 and 8 is that accuracy is generally not a function of the number of vehicles in the network. This is because GPS-only accuracy is largely based on the satellite geometry, which is limited for all vehicles such that adding more vehicles does not improve performance.

Before moving on, given that the $0^{\circ}$ road azimuth case is the worst-case scenario, it follows that this is when UWB and/or bearing data will be most important, that is, offer the most improvement. As such, for the rest of the paper, 

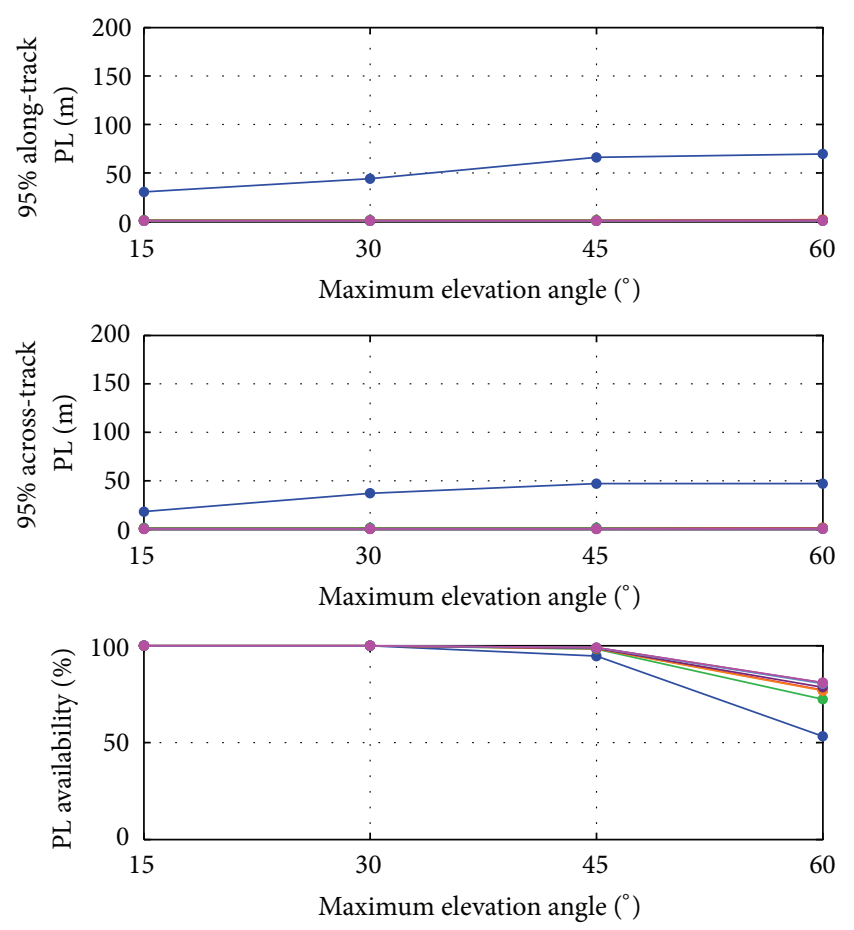

$\begin{array}{ll}\longrightarrow 2 \text { vehicles } & -6 \text { vehicles } \\ \longrightarrow 3 \text { vehicles } & \rightarrow 7 \text { vehicles } \\ \longrightarrow 4 \text { vehicles } & \rightarrow 8 \text { vehicles } \\ \longrightarrow 5 \text { vehicles } & \rightarrow 9 \text { vehicles }\end{array}$

FIGURE 12: 95th percentile along-track and across-track protection levels and protection level availability using GPS + UWB + bearing as a function of maximum elevation angle for a road azimuth of $0^{\circ}$.

only results for the $0^{\circ}$ road azimuth case are shown with the understanding that the $90^{\circ}$ road azimuth results are better.

Figure 9 shows the 95th percentile along- and acrosstrack accuracy when augmenting GPS with UWB or bearing data. When adding UWB data, a large improvement in the along-track accuracy is observed, which is a function of the distribution of the vehicles. Specifically, since the simulated vehicle network is primarily aligned in the alongtrack direction, it follows that this direction would benefit most from a UWB range measurement made in this direction. It is also interesting to note that the largest improvement comes with the addition of the third vehicle, which then forms a triangle of vehicles traveling together. The triangular shape provides better horizontal measurement geometry than in the linear (i.e., two-vehicle) case. By extension, the addition of subsequent vehicles provides smaller improvements because the overall improvement in geometry is less significant.

Adding bearing data results in a similar overall position accuracy improvement as when adding UWB data, but this time mainly in the across-track direction (instead of the along-track direction). The across-track improvement using bearing data is better than the along-track improvement due to UWB range because the assumed measurement accuracy of the bearing. Specifically, the "across-track ranging accuracy" for bearing is $0.5^{\circ} \times 15 \mathrm{~m}=0.17 \mathrm{~m}$ compared to $0.5 \mathrm{~m}$ for UWB in the along-track direction. The $15 \mathrm{~m}$ values used in the bearing calculation is the largest distance from any one vehicle to its nearest neighbour (actually $14.14 \mathrm{~m}$ ) and in many cases the distance is smaller resulting in an even smaller error. Finally, although not shown, including both UWB and bearing data yields horizontal solutions that are accurate to better than one metre, regardless of the elevation mask of the urban canyon. This is because a horizontal position solution can be determined using only the UWB and bearing measurements (which are accurate to $0.5 \mathrm{~m}$ for UWB and $0.5^{\circ}(\leq 0.17 \mathrm{~m})$ for bearing), and thus the solution is independent of the GPS masking environment.

4.3. Reliability. Similar to the accuracy results presented above, the reliability results are presented as 95th percentile values as a function of maximum elevation angle and number of vehicles in the network. The top two plots in Figure 10 respectively show the along- and across-track protection levels for the GPS-only case. The protection levels are generally insensitive to masking angles and tend to level off around $65 \mathrm{~m}$ for the along- and across-track directions. Given that the solution accuracies in Figure 7 are generally much worse than this, these results are counterintuitive. To understand this, the bottom plot in Figure 10 shows the "protection level availability;" that is, the percentage of the time that redundant observations are available such that reliability testing is possible. By extension, when the protection level is unavailable, this implies an infinite protection level. With this in mind, although the protection level values in Figure 10 remain approximately elevation angle independent, the number of available solutions where reliability testing is possible decreases rapidly with increasing elevation angle.

Similar plots for when UWB or bearing observations are used in the solution are shown in Figure 11. Compared to the GPS-only results above, three things are worth noting. First, adding either UWB or bearing observations improves the protection levels with UWB providing the most benefit in the along-track direction and bearing providing the most benefit in the across-track direction. Second, perhaps more important than the better reliability, there are also more solutions where redundant measurements are available (i.e., higher protection level availability). In other words, there are also fewer epochs (compared to the GPS-only case) where the protection levels are infinite. Third, the number of vehicles in the network also affects the protection levels. As more vehicles are added to the network, more observations are provided and thus the reliability improves. This suggests that larger networks of vehicles are more beneficial from a reliability perspective.

Finally, Figure 12 shows the reliability results when GPS, UWB, and bearing information are used together. As expected, the availability of redundant measurements is now much higher and is nearly $100 \%$ even for the $45^{\circ}$ elevation mask case. In the terms of the protection levels, whenever three or more vehicles are included in the network the protection levels are always less than $2 \mathrm{~m}$ in the along- 

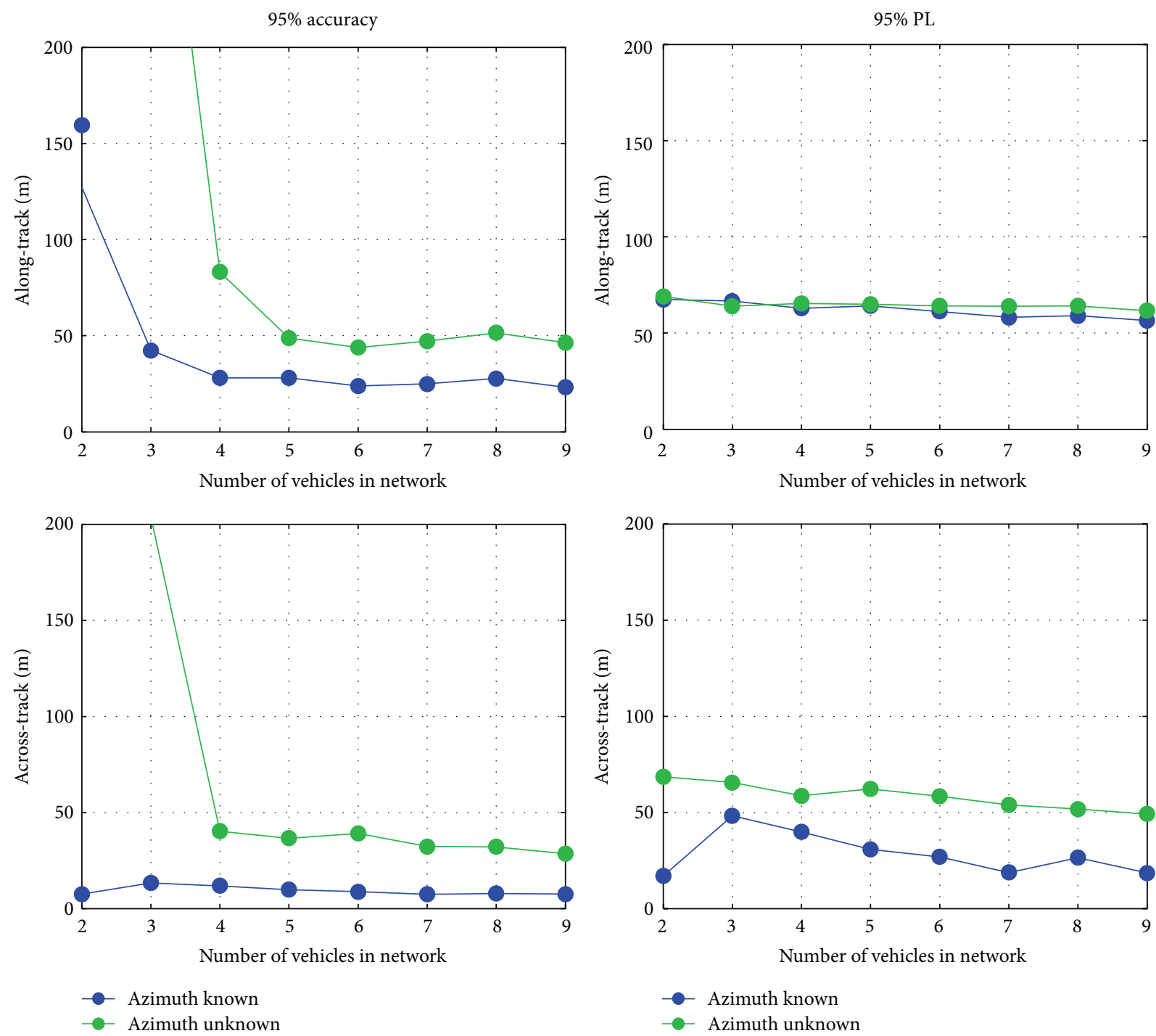

FIGURE 13: Effect of unknown vehicle azimuth on the 95th percentile accuracy (left) and protection levels (right) in the along-and across-track directions using GPS + bearing as a function of the number of vehicles in the network for a road azimuth of $0^{\circ}$.

and across-track directions. As mentioned above, this is because once there are three vehicles in the network, the UWB and bearing data can determine the horizontal solution without any GPS data and can serve as checks against each other. For the two-vehicle network, however, the UWB and bearing observations are still reliant on the lower-quality GPS observations to identify blunders. Correspondingly, the protection levels in this case are similar to the GPS-only values shown in Figure 10 but are available much more often.

4.4. Effect of Unknown Vehicle Azimuth. In the above results, it was assumed that the azimuth of any vehicle making a bearing measurement was known. In this section, the case where the azimuth(s) of bearing-measuring vehicle(s) need to be estimated is considered. Figure 13 shows the 95th percentile accuracy and protection levels when using GPS and bearing data as a function of the number of vehicles in the network. As expected, the solution accuracy is degraded compared to the case where the vehicle azimuth is known. This is especially true for the cases with three or fewer vehicles in the network. With five or more vehicles in the network, the accuracy is degraded by roughly a factor of two compared with the azimuth-known case.

For the reliability, the along-track performance is effectively the same regardless of whether the vehicle azimuth is known or not. This follows from the fact that bearing observations primarily affect the across-track solution. By extension, the across-track protection levels when the vehicle azimuth is unknown are observed to increase by a factor of approximately two compared to the azimuth-known case.

4.5. Effect of Kalman Filtering. All of the previous results were based on the use of a least-squares estimation algorithm even though most practical systems are expected to use some form of Kalman filtering. Therefore, some results obtained using a Kalman filter are shown here to give an idea of the 

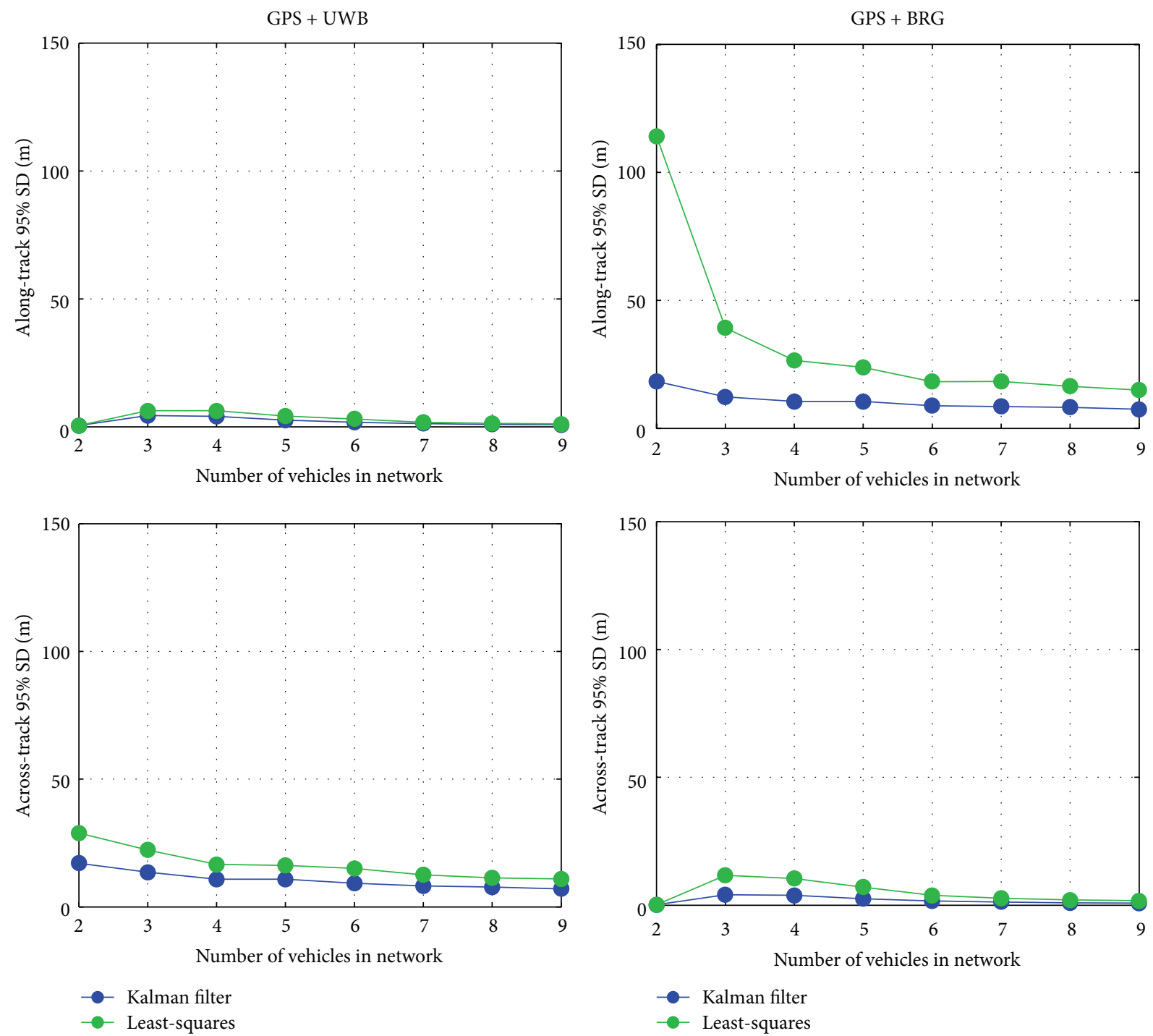

FIGURE 14: Least-squares and Kalman filter 95th percentile along- and across-track accuracy using GPS + UWB (left) and GPS + Bearing (right) for a $0^{\circ}$ road azimuth and a $45^{\circ}$ maximum elevation.

level of improvement that may be possible relative to the least-squares case. However, given the additional parameters/factors associated with Kalman filtering-primarily the type of stochastic model(s) selected and the level of process noise-a comprehensive assessment is beyond the scope of this paper. The results presented should therefore be interpreted as an indication of level of improvement that could be realized by using a Kalman filter. The Kalman filter model and corresponding parameters are summarized in Table 2 and are based on data collected in several field tests. All of the results in this section are based on a road azimuth of $0^{\circ}$ and a maximum elevation mask of $45^{\circ}$.

Although not shown, for the GPS-only case, the leastsquares accuracy (95th percentile) was always greater than $400 \mathrm{~m}$ regardless of the number of vehicles in the network. However, when a Kalman filter was used, the accuracy improved to approximately $25 \mathrm{~m}$. Accuracy results for the case where UWB or bearing data is used are shown in Figure 14. In this case, the use of a Kalman filter generally offers little improvement except in the along-track direction using two vehicles with GPS and bearing observations. In this particular situation, the bearing measurement only observes the across-track position because the vehicles are perfectly aligned in the direction of motion and as such, the Kalman filter's system model is able to provide considerable benefit in that direction.

In terms of reliability, the GPS-only results are largely unaffected when using a Kalman filter because of the relatively loose dynamics constraints in Table 2 . However, when adding UWB or bearing data, Figure 15 shows that fairly significant reductions in the protection levels are possible when using a Kalman filter, especially as the number of vehicles in the network increases. 
GPS + UWB
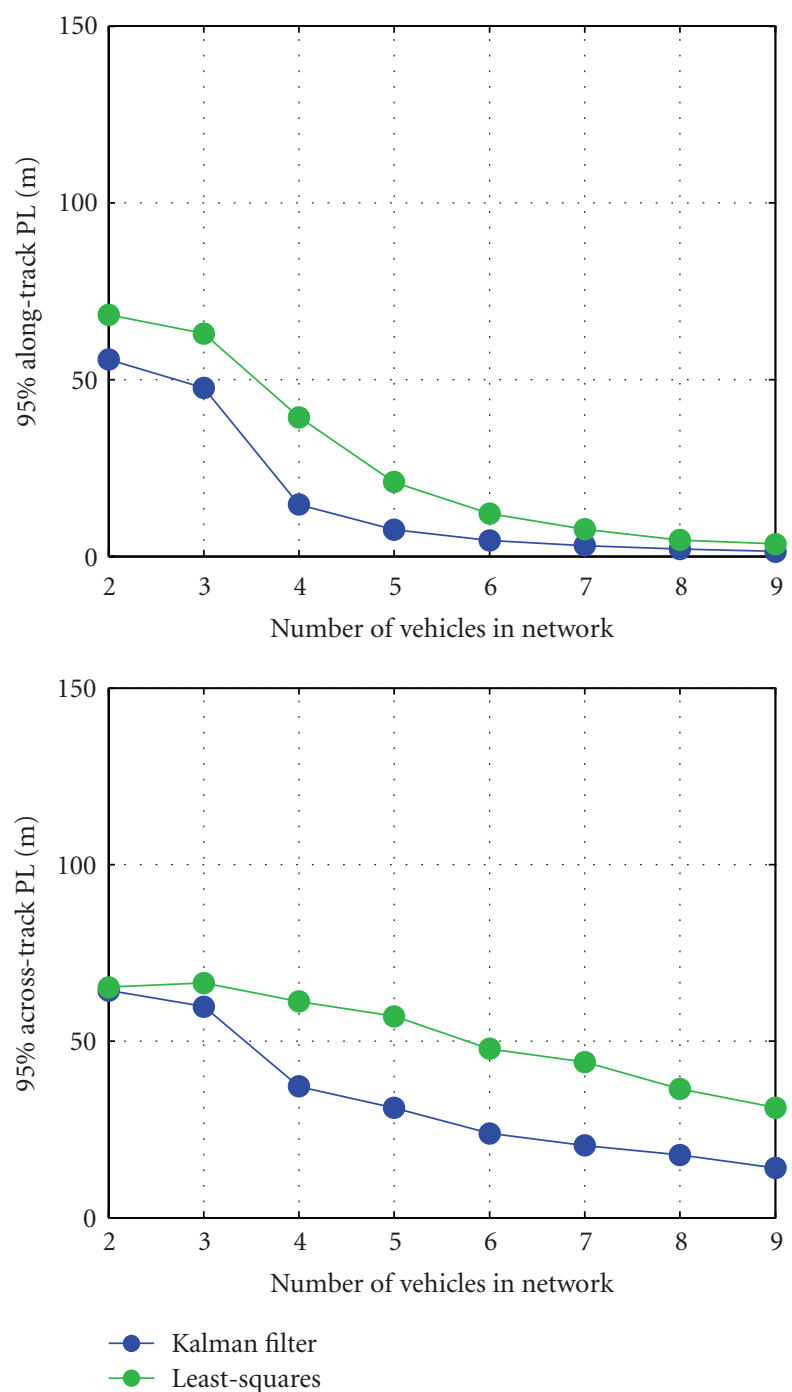

GPS + BRG
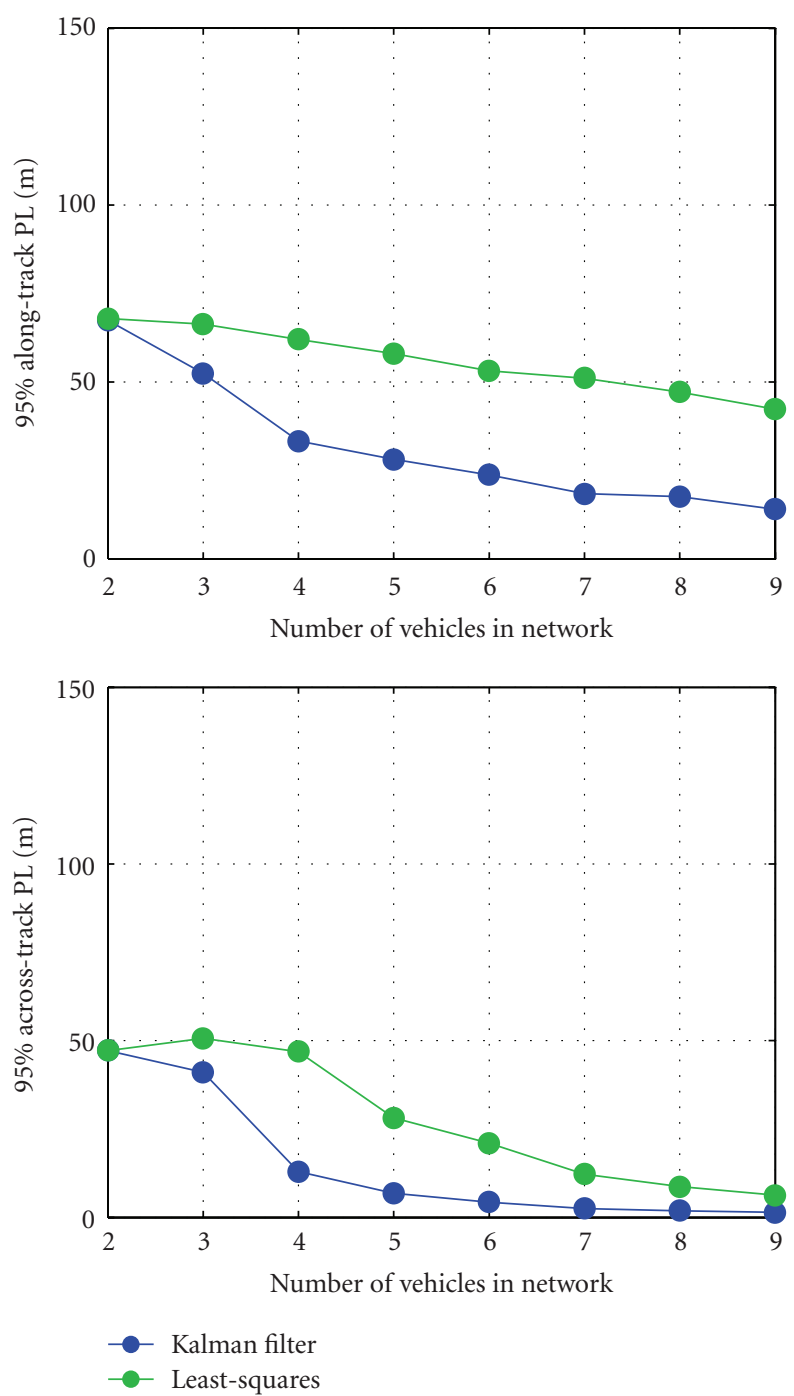

FIGURE 15: Least-squares and Kalman filter 95th percentile along- and across-track protection levels using GPS + UWB (left) and GPS + bearing (right) for a $0^{\circ}$ road azimuth and a $45^{\circ}$ maximum elevation.

TABLE 2: Kalman filter model parameters.

\begin{tabular}{lcr}
\hline State & Model & Parameters \\
\hline Position & Integrated velocity & N/A \\
GPS clock bias & Integrated clock drift & N/A \\
Horizontal velocity & First-order Gauss-Markov & $(\sigma=10 \mathrm{~m} / \mathrm{s}, \tau=2 \mathrm{~s})$ \\
Vertical Velocity & First-order Gauss-Markov & $(\sigma=1 \mathrm{~m} / \mathrm{s}, \tau=2 \mathrm{~s})$ \\
GPS clock drift & First-order Gauss-Markov & $(\sigma=1,000 \mathrm{~m} / \mathrm{s}, \tau=10 \mathrm{~s})$ \\
\hline
\end{tabular}

\section{Conclusions}

This paper investigated the effect of adding UWB range and bearing measurements to differential GPS as a means of determining the relative horizontal positions of various sizes of networks of moving vehicles.

The main conclusions that can be drawn from the results are as follows. (i) Solution availability benefits considerably from the inclusion of UWB and/or bearing data. In addition, larger benefits are observed as more vehicles are added to the network.

(ii) UWB measurements provide the largest improvement in the along-track direction while bearing measurements provide the largest improvement in the 
across-track direction. For scenarios with elevation mask angles $45^{\circ}$ or less, augmenting GPS with either sensor provides along- and across-track positioning accuracies (95th percentile) of less than $100 \mathrm{~m}$ while the GPS-only solution often exceeds $200 \mathrm{~m}$. When both UWB and bearing data are used, the solution accuracy was always at the metre level due to the high quality of these measurements and the fact that a range and bearing can determine the relative horizontal position without and GPS data at all.

(iii) Although the GPS-only protection levels were reasonable, the number of epochs where reliability checking was possible in the simulated urban canyon was highly compromised. Including UWB or bearing observations not only improved the protection levels, but also provided a much higher probability of having a redundant set of observations. In addition, using either sensor with GPS showed that reliably continued to improve as more vehicles were added to the network.

(iv) When GPS was augmented with both UWB and bearing data, as long as there are at least three vehicles in the network, the protection levels are below $2 \mathrm{~m}$ and the availability of redundant solutions is nearly $100 \%$ even with a GPS elevation mask angle of $45^{\circ}$.

(v) If the azimuth of bearing-observing vehicles needs to be estimated, the accuracy and reliability of the solution decreased by approximately a factor of two.

This paper presents covariance simulation results only. Preliminary field tests have been conducted to test a three-vehicle V2V scenario [25]. More extensive field tests of a prototype system are planned for the Summer 2012.

\section{Acknowledgments}

The research presented in this paper was conducted as part of a collaborative research and development grant between the University of Calgary, General Motors of Canada, and Natural Sciences and Engineering Research Council of Canada. Additional funding was also provided by Alberta Innovates Technology Futures (formerly Alberta Ingenuity Fund).

\section{References}

[1] N. Luo and G. Lachapelle, "Relative positioning of multiple moving platforms using GPS," IEEE Transactions on Aerospace and Electronic Systems, vol. 39, no. 3, pp. 936-948, 2003.

[2] C. Basnayake, "Communication on the road," GPS World, pp. 28-36, 2009.

[3] S. Savasta, M. Pini, and G. Marfia, "Performance assessment of a commercial gps receiver for networking applications," in Proceedings of the 5th IEEE Consumer Communications and Networking Conference (CCNC '08), pp. 613-617, January 2008.

[4] J. Stephen and G. Lachapelle, "Development and testing of a GPS-augmented multi-sensor vehicle navigation system," Journal of Navigation, vol. 54, no. 2, pp. 297-319, 2001.

[5] R. Cardinali, L. De Nardis, M.-G. D. Benedetto, and P. Lombarde, "UWB ranging accuracy in high- and low-data-rate applications," IEEE Transactions on Microwave Theory and Techniques, vol. 54, no. 4, pp. 1865-1875, 2006.

[6] R. Fontana, "Experimental results from an ultra wideband precision geolocation system," in Ultra-Wideband, Short-Pulse Electromagnetics 5, pp. 215-223, Kluwer Academic Publishers, 2002.

[7] G. MacGougan, K. O’Keefe, and R. Klukas, “Tightly-coupled GPS/UWB integration," Journal of Navigation, vol. 63, no. 1, pp. $1-22,2010$.

[8] G. MacGougan, K. O’Keefe, and R. Klukas, "Accuracy and reliability of tightly coupled GPS/ultra-wideband positioning for surveying in urban environments," GPS Solutions, vol. 14, no. 4, pp. 351-364, 2010.

[9] E. Mok, L. Xia, R. Guenther, and H. Tian, "A case study on the feasibility and performance of an UWB-AoA real time location system for resources management of civil construction projects," Journal of Applied Geodesy, vol. 4, pp. 23-32, 2010.

[10] P. J. Buist, P. J. G. Teunissen, G. Giorgi, and S. Verhagen, "Multivariate bootstrapped relative positioning of spacecraft using GPS L1/Galileo E1 signals," Advances in Space Research, vol. 47 , no. 5, pp. 770-785, 2011.

[11] K. O’Keefe, M. G. Petovello, G. Lachapelle, and M. E. Cannon, "Assessing probability of correct ambiguity resolution in the presence of time-correlated errors," Navigation, vol. 53, no. 4, pp. 269-282, 2006.

[12] A. Leick, GPS Satellite Surveying, John Wiley \& Sons, Hoboken, NJ, USA, 3rd edition, 2004.

[13] J. Klobuchar, "Ionospheric effect on GPS," in Global PositionIng System: Theory and Applications, B. W. Parkinson and J. J. Spilker Jr., Eds., vol. 1, American Insistute of Aeronautics and Astronautics, Washington, DC, USA, 1996.

[14] A. Leick, GPS Satellite Surveying, John Wiley \& Sons, 3rd edition, 2004.

[15] G. MacGougan, K. O’Keefe, and R. Klukas, "Ultra-wideband ranging precision and accuracy," Measurement Science and Technology, vol. 20, no. 9, Article ID 095105, 2009.

[16] G. MacGougan, K. O’Keefe, and R. Klukas, “Tightly-coupled GPS/UWB integration,” Journal of Navigation, vol. 63, no. 1, pp. $1-22,2010$.

[17] C. Tang, Accuracy and Reliability of Various DGPS Approaches [M.S. thesis], Geomatics Engineering, University of Calgary, Calgary, Canada, 1996.

[18] R. G. Brown and P. Y. C. Hwang, Introduction to Random Signals and Applied Kalman Filtering, John Wiley \& Sons, 3rd edition, 1997.

[19] A. Gelb, Ed., Applied Optimal Estimation, The MIT Press, Cambridge, Mass, USA, 1974.

[20] K.-R. Koch, Parameter Estimation and Hypothesis Testing in Linear Models, Springer, Berlin, Germany, 1999.

[21] A. Saalfeld, "Generating basis sets of double differences," Journal of Geodesy, vol. 73, no. 6, pp. 291-297, 1999.

[22] AutonomousStuff, "RADAR Product Catalog," 2012, http:// www.autonomoustuff.com/uploads/9/6/0/5/9605198/__radar_ product_catalog_v2.pdf.

[23] FRP, "Federal Radionavigation Plan," ed: U.S. Departments of Defense, Homeland Security, and Transport, 2008.

[24] P. J. G. Teunissen and M. A. Salzmann, "A recursive slippage test for use in state-space filtering," Manuscripta Geodaetica, vol. 14, no. 6, pp. 383-390, 1989. 
[25] M. G. Petovello, K. O'Keefe, B. Chan, S. Spiller, C. Pedrosa, and C. Basnayake, "Demonstration of inter-vehicle UWB ranging to augment DGPS for improved relative positioning," in Proceedings of the 23rd International Technical Meeting of the Satellite Division of the Institute of Navigation 2010 (ION GNSS '10), pp. 1198-1209, Portland, Ore, USA, September 2010. 

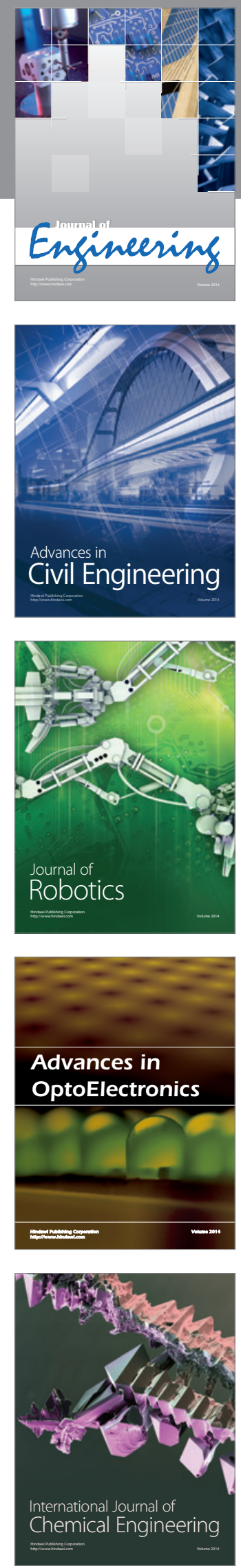

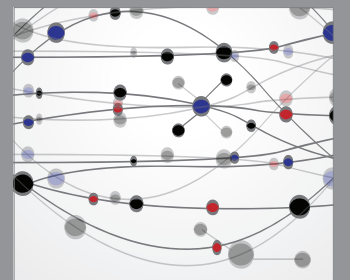

The Scientific World Journal
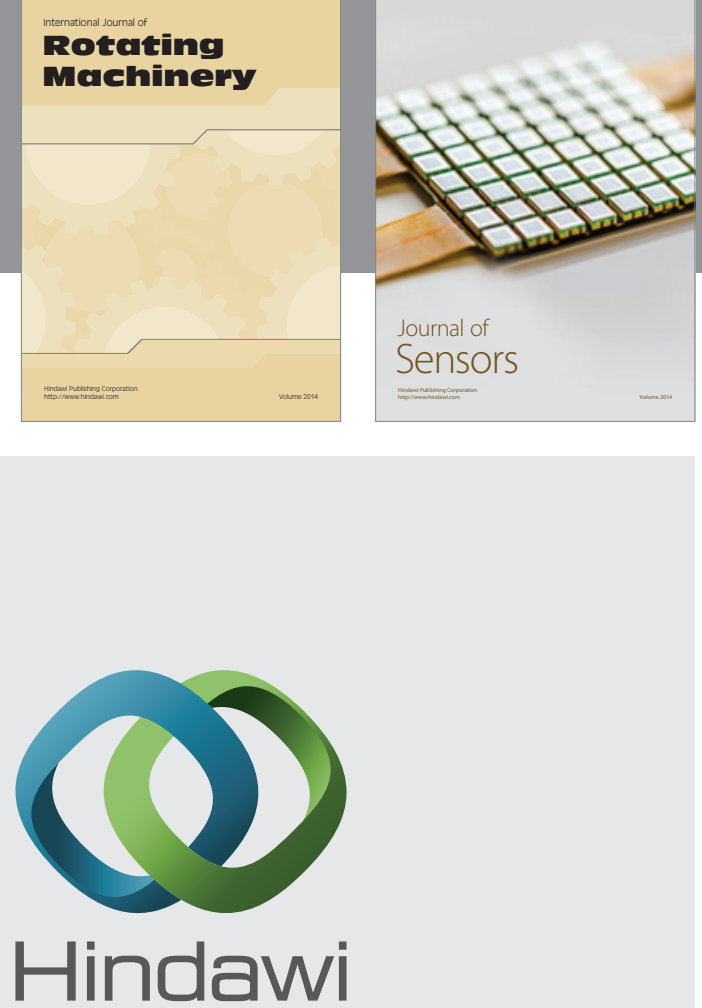

Submit your manuscripts at http://www.hindawi.com
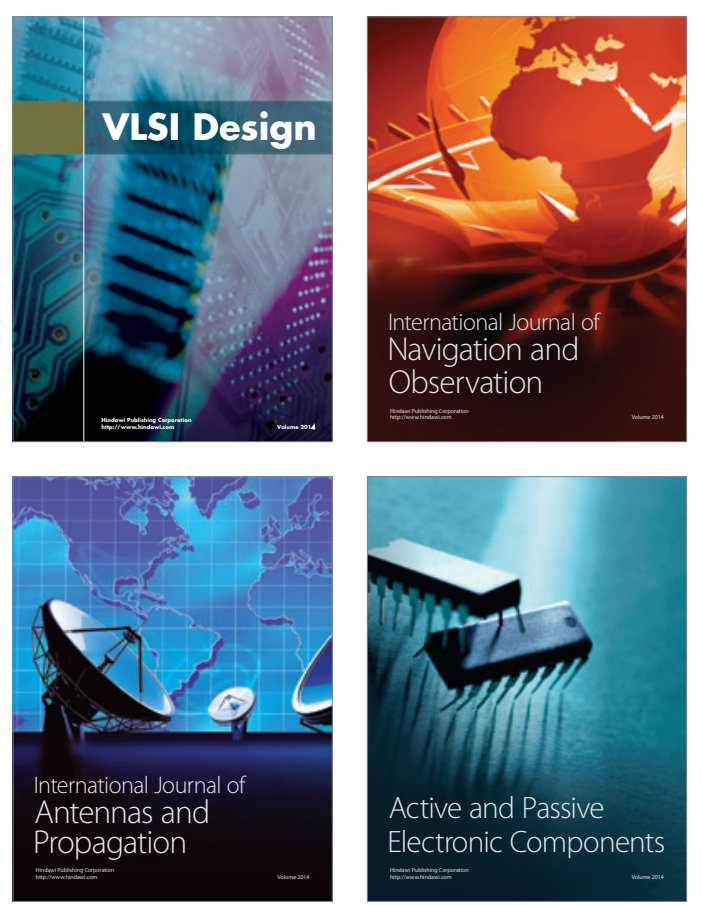
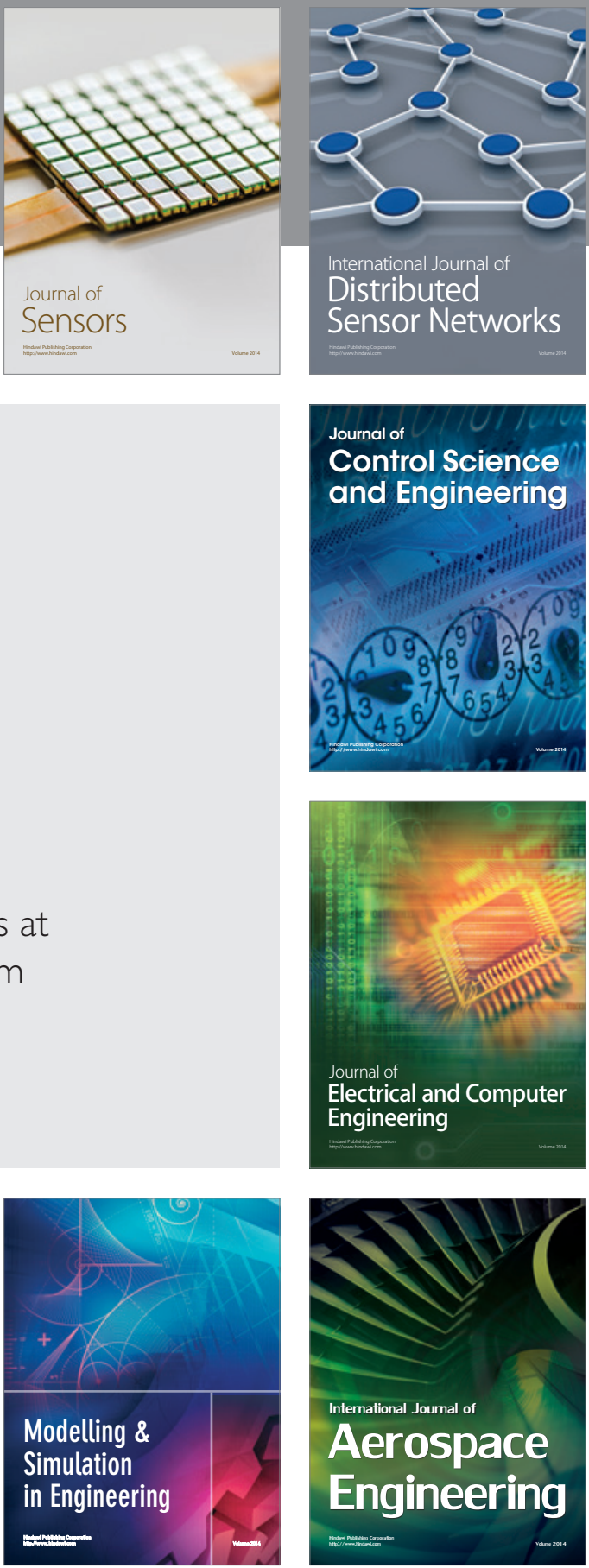

Journal of

Control Science

and Engineering
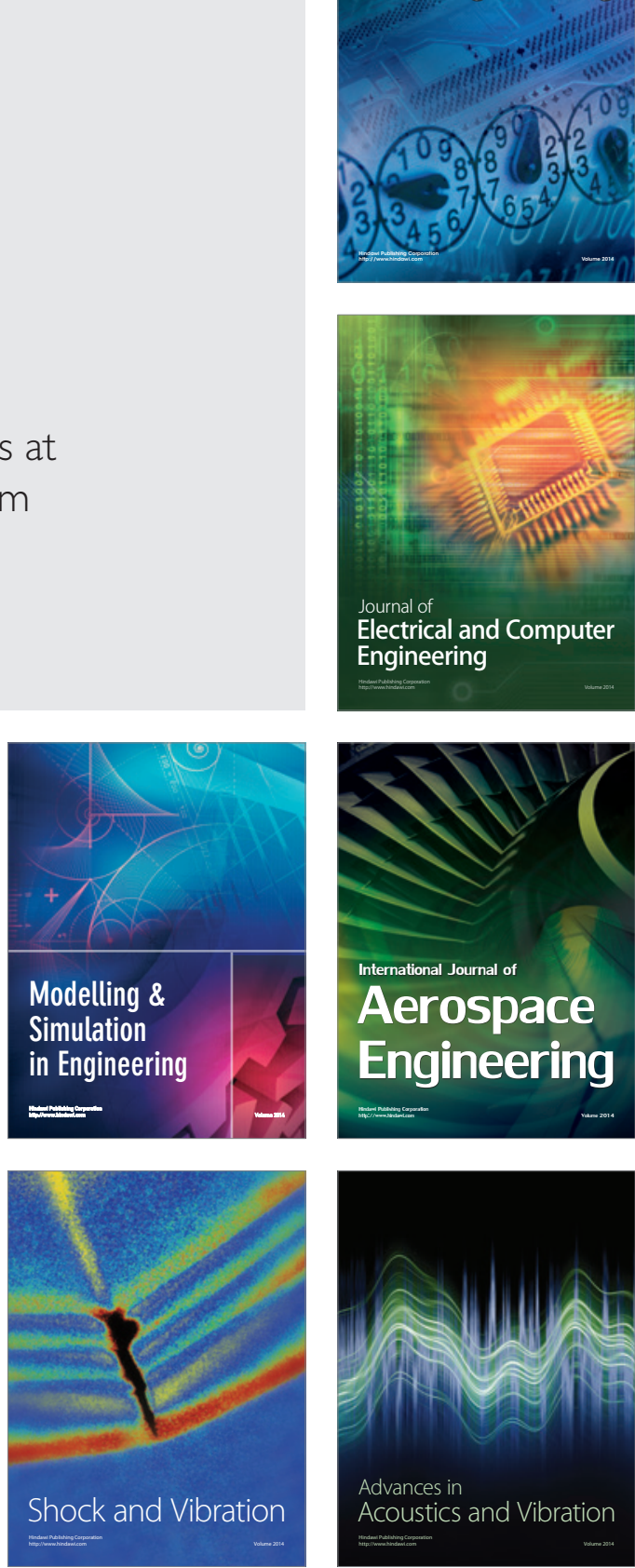